\title{
Caracterização do atendimento antirrábico humano em Jataí, Goiás, no ano de 2014
}

\author{
Characterization of human anti-rabies treatment in Jataí, Goiás, in 2014 \\ Caracterización de la atención antirrábica humana en Jataí, Goiás, en 2014
}

Recebido: 26/01/2021 | Revisado: 01/02/2021 | Aceito: 02/02/2021 | Publicado: 07/02/2021

Dionatan Assis de Azevedo

ORCID: https://orcid.org/0000-0003-4167-2463

Universidade Nove de Julho, Brasil

E-mail: dionatanazevedo@yahoo.com.br

Andressa Vaz Martins

ORCID: https://orcid.org/0000-0002-8530-7719

Universidade Federal de Jataí, Brasil

E-mail: andressavaz@discente.ufg.br

Dirceu Guilherme de Souza Ramos

ORCID: https://orcid.org/0000-0001-9603-6638

Universidade Federal de Jataí, Brasil

E-mail: dgramos_vet@ufg.br

Klaus Casaro Saturnino

ORCID: https://orcid.org/0000-0001-8493-8669

Universidade Federal de Jataí, Brasil

E-mail: klaus.sat@ufg.br

Andreia Vitor Couto do Amaral

ORCID: https://orcid.org/0000-0001-6406-2372

Universidade Federal de Jataí, Brasil

E-mail: andreiavcvet@ufg.br

Alana Flávia Romani

ORCID: https://orcid.org/0000-0002-8138-408X Universidade Federal de Jataí, Brasil

E-mail: alana_romani@ufg.br

Ísis Assis Braga

ORCID: https://orcid.org/0000-0001-5713-4833

Centro Universitário de Mineiros, Brasil

E-mail: isis@unifimes.edu.br

Carolina de Alvarenga Cruz

ORCID: https://orcid.org/0000-0002-1623-8932

Médica Veterinária Autônoma, Brasil E-mail: carol_a_cruz@yahoo.com.br

Daniel Bartoli de Sousa

ORCID: https://orcid.org/0000-0003-3209-7911 Universidade Federal de Jataí, Brasil

E-mail: daniel_bartoli_sousa@ufg.br

Raphaella Barbosa Meirelles-Bartoli ORCID: https://orcid.org/0000-0002-7147-5711 Universidade Federal de Jataí, Brasil E-mail: raphaella@ufg.br

\begin{abstract}
Resumo
O objetivo deste estudo foi caracterizar o perfil epidemiológico do atendimento antirrábico humano pré e pósexposição, no município de Jataí - Goiás, no ano de 2014. Para tal, realizou-se um estudo descritivo referente aos atendimentos antirrábicos humanos inseridos no Sistema de Informação de Agravos de Notificação (SINAN). Esses dados foram tabulados e analisados em planilhas do software Microsoft Office Excel 2010. Ocorreram 563 atendimentos antirrábicos humano em 2014, com grande maioria residente na zona urbana $(98,4 \%)$. Do total de pacientes, $502(89,16 \%)$ foram em busca de profilaxia pós-exposição, sendo que destes agravos, o principal tipo de exposição foi mordedura (96\%), em mãos/pés (39,24\%), com 83,07\% dos acidentes com ferimentos únicos, e predomínio de lesões profundas $(90,64 \%)$. Em relação ao animal agressor, a espécie mais frequente foi a canina (77,79\%), 86,32\% dos animais apresentaram-se sadios no momento do acidente, e houve a observação de $81,27 \%$ dos animais. A modalidade de tratamento mais indicada foi a observação do animal associada ao uso de vacina (78\%), contudo, a efetivação da profilaxia prescrita ocorreu em apenas 83,30\% dos casos. Conclui-se que o estudo contribuiu para a caracterização e entendimento do perfil epidemiológico da profilaxia antirrábica humana no município, evidenciando a importância da educação em saúde, da qualificação técnica dos profissionais que prestam o atendimento, e necessidade constante das ações de vigilância epidemiológica.
\end{abstract}


Palavras-chave: Epidemiologia; Profilaxia; Raiva; Saúde pública; Vacinas antirrábicas.

\begin{abstract}
The aim of this study was to characterize the epidemiological profile of human anti-rabies treatment pre and postexposure, in the city of Jataí, Goiás, in 2014. For this, a descriptive study was carried out regarding the human antirabies treatment included in the Notifiable Diseases Information System. These data were tabulated and analyzed in Microsoft Office Excel 2010 spreadsheets. There were 563 of human anti-rabies treatments in 2014, with a large majority residing in the urban area $(98.4 \%)$. Of the total number of patients, $502(89.16 \%)$ were in search of postexposure prophylaxis, and of these injuries, the main type of exposure was bite (96\%), hands / feet (39.24\%), with $83.07 \%$ of single injuries accidents, and a predominance of deep wounds (90.64\%). Regarding the aggressor animal, the most frequent species was canine $(77.79 \%), 86.32 \%$ of the animals were healthy at the time of the accident, and $81.27 \%$ of them were observed. The most indicated treatment modality was the observation of the animal associated with the use of vaccine (78\%), however, the effectiveness of the prescribed prophylaxis occurred in only $83.30 \%$ of the cases. It is concluded that the study contributed to the characterization and understanding of the epidemiological profile of human anti-rabies prophylaxis in the municipality, showing the importance of health education, the technical qualification of the professionals who provide care, and the constant necessity of epidemiological surveillance actions.
\end{abstract}

Keywords: Epidemiology; Prophylaxis; Public health; Rabies; Rabies vaccines.

\title{
Resumen
}

El objetivo de este estudio fue caracterizar el perfil epidemiológico de la atención pre y posexposición antirrábica humana, en la ciudad de Jataí - Goiás, en 2014. Para ello, se realizó un estudio descriptivo sobre la antirrábica humana atención incluida en el Sistema de Información de Enfermedades Notificables (SINAN). Estos datos se tabularon y analizaron en hojas de cálculo de Microsoft Office Excel 2010. Hubo 563 llamadas de personas contra la rabia en 2014, con una gran mayoría residentes en el área urbana $(98,4 \%)$. Del total de pacientes, $502(89,16 \%)$ estaban en busca de profilaxis posexposición, y de estas lesiones, el principal tipo de exposición fue mordedura (96\%), manos / pies $(39,24 \%)$, con $83,07 \%$ de accidentes con traumatismo único, y predominio de traumatismos profundos $(90,64 \%)$. En cuanto al animal agresor, la especie más frecuente fue el canino (77,79\%), el 86,32\% de los animales estaban sanos al momento del accidente y se observó el $81,27 \%$ de los animales. La modalidad de tratamiento más indicada fue la observación del animal asociada al uso de vacuna (78\%), sin embargo, la efectividad de la profilaxis prescrita se presentó en solo el $83.30 \%$ de los casos. Se concluye que el estudio contribuyó a la caracterización y comprensión del perfil epidemiológico de la profilaxis antirrábica humana en el municipio, destacando la importancia de la educación en salud, la calificación técnica de los profesionales que brindan atención y la necesidad constante de vigilancia epidemiológica.

Palabras clave: Epidemiología; Profilaxis, Rabia; Salud pública; Vacunas antirrábicas.

\section{Introdução}

A raiva é uma importante zoonose caracterizada por encefalite viral progressiva aguda, causada por um vírus da família Rhabdoviridae e do gênero Lyssavirus (Brasil, 2014). A doença é considerada como a primeira enfermidade que os animais poderiam transmitir aos seres humanos (Gomide-Junior, 2013). Apresenta ampla distribuição geográfica, taxa de letalidade próxima a 100\% e alto impacto na saúde pública (Rocha, 2017).

O tratamento específico para a doença não existe, portanto, em caso de suspeita de exposição ao vírus rábico, a profilaxia pós-exposição deve ser rigorosamente cumprida. A anamnese completa é de muito importante e deve utilizar-se da Ficha de Atendimento Antirrábico Humano para obter todas as informações sobre o caso. Além disso, deve classificar o acidente em leve ou grave, dependendo das características do ferimento, como localização da ferida, profundidade, extensão e o número de lesões, além da possibilidade de observação do animal agressor por dez dias, procedência e hábitos de vida, se ocorrido envolvendo cães e gatos (Brasil, 2014).

Mediante esse atendimento, é possível indicar corretamente o tratamento profilático ao paciente (Brasil, 2017), uma vez que a profilaxia de seres humanos expostos ao risco de infecção pelo vírus rábico ainda pode incluir ocorrência de reações adversas pós-vacinais indesejáveis, tanto de ordem local, quanto geral (Garcia, 1999). Já a profilaxia pré-exposição deve ser realizada por meio de vacinas em pessoas que possuem risco de exposição permanente ao vírus da raiva, de acordo com o desenvolvimento de suas atividades ocupacionais (Brasil, 2014). 
Devido a variedade de ciclos (urbano, silvestre ou rural) e das consequências irreversíveis aos humanos, a vigilância epidemiológica da raiva é obrigatória. O conhecimento de dados epidemiológicos e adoção de medidas de bloqueio de foco e de controle animal tanto por parte do serviço de saúde quanto do serviço de controle de zoonoses é fundamental para que ocorra a correta profilaxia em tempo oportuno (Veloso et al., 2011a).

Diante do exposto, presente trabalho tem por objetivo caracterizar o perfil epidemiológico do atendimento antirrábico humano pré e pós-exposição, e analisar a adequação de sua indicação no município de Jataí, Goiás, no ano de 2014, visando a obtenção de subsídios para o aprimoramento dos atendimentos, e consequente redução de risco de transmissão da raiva humana.

\section{Metodologia}

A pesquisa baseou-se na realização de estudo descritivo, por meio de levantamento de dados registrados na Ficha de Atendimento Antirrábico Humano, inseridos no Sistema de Informações de Agravos de Notificação (SINAN), de pacientes atendidos em Jataí, GO, no ano de 2014, para efeito de profilaxia antirrábica pré e pós-exposição, excluindo àqueles que não residiam neste município.

Para a presente investigação, foram utilizadas as seguintes variáveis de estudo: local de residência do indivíduo (zona rural ou urbana), tipo de exposição ao vírus rábico, localização do agravo, tipo de ferimento, quantidade de ferimentos, possibilidade de antecedentes de tratamento antirrábico, espécie do animal agressor, condição do animal para fins de conduta do tratamento, tratamento indicado, condição final do animal (após período de observação), interrupção do tratamento e qual o motivo. Esses dados foram tabulados e analisados em planilhas do software Microsoft Office Excel 2010.

Como foram utilizados para o presente estudo apenas dados secundários, não nominais, e de acesso público, não há necessidade de apreciação por Comitê de Ética em Pesquisa, conforme define a Resolução do Conselho Nacional de Saúde (CNS/MS) nº 510, de 7 de abril de 2016.

\section{Resultados}

No ano de 2014, foram atendidos 563 pacientes em busca de tratamento profilático antirrábico em Jataí, Goiás. Como demonstrado na Figura 1, foi o ano em que ocorreu maior número de atendimentos quando comparado aos anos anteriores, desde 2009, sendo o menor número em 2010, com 315 atendimentos.

Figura 1. Número de atendimentos de profilaxia antirrábica pré e pós exposição, entre os anos 2009 e 2014, em Jataí, Goiás.

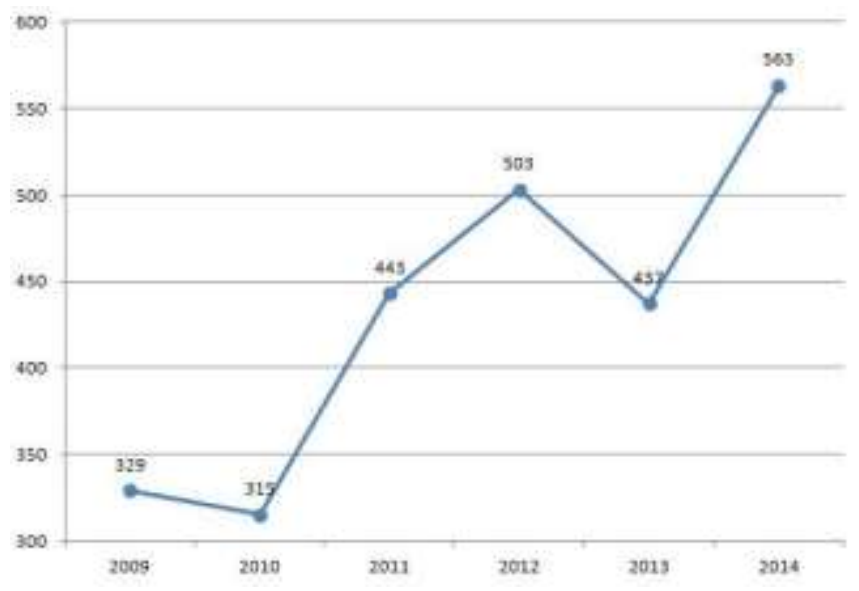

Fonte: Autores. 
Em relação ao registro do local de residência, dos 562 pacientes em que essa informação foi registrada, verifica-se que a maioria reside na zona urbana $(98,40 \%)$, uma pequena parte na zona rural $(1,42 \%)$, nenhum percentual na área de transição entre a cidade e o campo (periurbana) e uma ficha $(0,17 \%)$ com falha de preenchimento.

Do total de 563 atendimentos, 61 (10,83\%) realizaram atendimentos para profilaxia antirrábica pré-exposição graças a um programa de Imunização e Controle Sorológico Antirrábico Humano e Animal realizado anualmente com alunos de uma Universidade Federal localizada em Jataí, ou seja, não sofreram agressão por nenhum tipo de animal.

Os demais 502 pacientes $(89,16 \%)$ que procuraram atendimento antirrábico pós-exposição tiveram os agravos caracterizados, como descrito na Tabela 1. Destes, o tipo de exposição relatada mais comum foi a mordedura, representando 96\% dos atendimentos, seguida por arranhadura (2,98\%) e lambedura $(0,99 \%)$, não havendo exposição por contato indireto.

Tabela 1. Caracterização dos agravos dos atendimentos antirrábicos pós-exposição, de acordo com tipo de exposição ao vírus rábico, localização da lesão, tipo de ferimento, e profundidade, em Jataí, GO, no ano de 2014 (N=502).

\begin{tabular}{lll} 
Variáveis & N & \% \\
\hline Tipo de Exposição & 482 & $96,00 \%$ \\
\hline Mordedura & 15 & $2,98 \%$ \\
Arranhadura & 5 & $0,99 \%$ \\
Lambedura & 0 & - \\
Contato Indireto & 0 & - \\
Outros & & \\
\hline Localização & 197 & $39,24 \%$ \\
\hline Mãos ou pés & 167 & $33,27 \%$ \\
Membros inferiores & 74 & $14,74 \%$ \\
Membros superiores & 43 & $8,57 \%$ \\
Cabeça ou Pescoço & 12 & $2,39 \%$ \\
Mucosa & 9 & $1,79 \%$ \\
Tronco & & \\
\hline Tipo de Ferimento & 417 & $83,07 \%$ \\
\hline Único & 30 & $5,98 \%$ \\
Múltiplo & 55 & $10,96 \%$ \\
Fichas Incompletas & & \\
\hline Profundidade & 455 & $90,64 \%$ \\
\hline Profundo & 43 & $8,57 \%$ \\
Superficial & 4 & $0,80 \%$ \\
Dilacerante & & \\
\hline & & \\
\hline & & \\
\hline
\end{tabular}

Fonte: Autores.

Quanto a localização específica da lesão, as mãos e pés foram as regiões mais atingidas $(39,24 \%)$, seguidas por outras regiões dos membros inferiores $(33,27 \%)$ e superiores $(14,74 \%)$. As localizações menos comuns relatadas foram cabeça ou pescoço $(8,57 \%)$, mucosa $(2,39 \%)$ e tronco $(1,79 \%)$. Em 83,07\% dos agravos, os pacientes tiveram ferimentos únicos, 5,98\% ferimentos múltiplos e 10,96\% das fichas apresentaram erro ou falha no campo de preenchimento. Em 
relação a profundidade da lesão, a maioria dos casos apresentou ferimentos profundos $(90,64 \%)$, seguido por 8,57\% de lesões superficiais, com raras lesões dilacerantes $(0,8 \%)$.

Destes pacientes que passaram por atendimento antirrábico pós-exposição, 98,4\% deles nunca haviam recebido tratamento antirrábico. Antecedente de aplicação de vacina em tratamento pré-exposição foi verificada em $0,53 \%$ dos pacientes, enquanto que 1,07\% já haviam recebido tratamento pós-exposição, ou seja, um total de 1,6\% de pacientes com exposição prévia à profilaxia antirrábica. Em uma das fichas este campo não foi preenchido, não sendo possível afirmar se esse paciente recebeu algum dia este tipo de tratamento.

Em relação ao animal agressor envolvido, a maioria dos pacientes $(77,79 \%)$, foi atacada por cães. Os felinos domésticos representaram a segunda espécie, com 9,2\% das ocorrências. Proporções reduzidas foram atribuídas a quirópteros $(0,71 \%)$, primatas $(0,17 \%)$ e herbívoros domésticos $(0,17 \%)$. A informação não foi preenchida em 67 casos $(11,90 \%)$. A avaliação da condição de saúde do animal agressor no momento do acidente se deu em 490 fichas de atendimento. Nestas, 423 animais $(86,32 \%)$ apresentaram-se sadios no momento da ocorrência; desapareceram ou morreram 58 animais $(11,83 \%)$, e um total de $9(1,8 \%)$ animais foram relatados como suspeitos de raiva no momento da agressão. Entretanto, nenhum animal raivoso foi descrito durante as ocorrências que motivou o atendimento. Houve a observação de 408 animais $(81,27 \%)$ para constatação de sua condição final, e desses, 402 (80\%) finalizaram o período de 10 dias sem nenhuma alteração de comportamento ou óbito; 6 animais morreram $(1,2 \%)$ e tiveram amostras encaminhadas ao laboratório, com todos negativos para raiva. Em 94 atendimentos (18,72\%), não houve acompanhamento dos animais envolvidos no agravo.

Considerando a modalidade de tratamento indicado, a observação do animal associada ao uso de vacina foi indicada para 392 pacientes (78\%), seguida de soro e vacina para 67 (13,3\%) indivíduos, observação do animal apenas, em $27(5,3 \%)$ dos casos, e somente vacina em $12(2,4 \%)$ casos. Em 4 atendimentos $(0,8 \%)$ não houve registro de informação.

Dos 563 pacientes para os quais foi prescrito tratamento profilático (incluindo pré e pós-exposição), ocorreu a sua efetiva aplicação em 469 (83,30\%). A interrupção de esquema aconteceu em 8 pacientes (1,42\%), sendo que desses, 5 pacientes $(0,88 \%)$ o fizeram por indicação do Serviço de Saúde, e $3(0,53 \%)$ interromperam por abandono. Essa informação foi negligenciada em $15,27 \%$ das profilaxias, que corresponde a 86 fichas de atendimento.

\section{Discussão}

O pico de atendimentos em Jataí, entre os anos 2009 a 2014, ocorreu neste último ano, e a procura pelos atendimentos de profilaxia antirrábica apresentou um aumento significativo a partir do ano de 2011, que possivelmente ocorreu como reflexo da inserção do Núcleo de Imunização e Controle Sorológico Antirrábico Humano e Animal na Universidade Federal do município, levando alunos recém ingressados na Universidade, alunos veteranos, bem como professores e outros profissionais expostos de forma permanente ao vírus rábico, a procurarem atendimento médico e realizarem o esquema profilático préexposição contra a raiva.

O fato de que $98,4 \%$ dos atendimentos terem sido de pacientes que residem na zona urbana, demonstra a distribuição da população no território municipal, e foi de acordo com o encontrado por outros autores (Carvalho et al., 2002; Frias, 2008; Filgueira et al., 2011; Müller et al., 2011; Gomide-Junior, 2013). Frias (2008) analisou que os acidentes na zona urbana, na grande maioria, ocorrem em residências envolvendo animais domésticos e geralmente vacinados contra a raiva, e por esse motivo, esses animais podem ser considerados de baixo risco para a transmissão da doença.

Em relação às fichas de atendimento antirrábico pós-exposição, o tipo de exposição mais frequente no município foi a mordedura (96\%), corroborando com outros estudos (Carvalho et al., 2002; Filgueira et al., 2011; Veloso et al., 2011b; Frias et al., 2012; Gomide-Junior, 2013; Benedetti, Capistrano, Borges \& Vieira Filho, 2020). Uma justificativa para a mordedura ser 
mais frequente, se deve ao fato do paciente se atentar em relatar o acidente pela gravidade da lesão e à possibilidade de esta estar relacionada com a raiva, diferentemente do que a população considera para a arranhadura e lambedura de um animal (Frias et al., 2012; Gomide-Junior, 2013).

Quanto as localizações de lesão corporal, o predomínio de mãos ou pés, também refletem os achados de outros trabalhos (Filgueira et al., 2011; Frias et al., 2012; Gomide-Junior, 2013; Silva et al., 2013). De acordo com Filgueira et al. (2011), Gomide-Junior (2013) e Silva et al. (2013), quando a ataque ocorre, essas regiões estão mais ao alcance dos animais, e por serem utilizadas como defesa à agressão, aumentam a chance de serem mais comprometidas durante o ataque. Já em relação a segunda localização mais comum, no presente estudo foram membros inferiores, diferentemente do trabalho de Gomide-Junior (2013), que demonstrou cabeça e pescoço como a segunda localização mais afetada, justificando o resultado devido a estatura das vítimas e à tentativa de defesa, por parte do cão, em brincadeiras interpretadas como provocativas.

O predomínio de lesões profundas $(90,6 \%)$ no presente estudo foi encontrado também por Benedetti et al. (2020), com 46,8\% dos agravos analisados com ferimentos profundos, mas não está de acordo com resultados encontrados por outros autores, que demonstram maior percentual de lesões superficiais (Carvalho et al., 2002; Fortes et al., 2007; Frias et al., 2012; Gomide-Junior, 2013). De acordo com Fortes et al. (2007), os ferimentos, quando superficiais, não foram gerados por comportamentos agressivos intenso e/ou contínuo dos animais envolvidos, situação contrária observada em Jataí, onde 90,6\% dos atendimentos tiveram lesões profundas. Essa informação, principalmente por apresentar proporção muito superior ao único estudo encontrado com predomínio de ferimento profundo, pode indicar falha na identificação de lesões superficiais e profundas por parte da equipe responsável pelo atendimento às profilaxias antirrábica pós-exposição.

A Ficha de Atendimento Antirrábico Humano dispõe do campo com informação sobre tratamento antirrábico antecedente, que é de extrema importância para a decisão do profissional da saúde em relação a instituição da nova profilaxia. Do total de atendimentos em Jataí, 1,6\% já haviam recebido tratamento prévio de profilaxia antirrábica, número bem inferior ao de Garcia et al. (1999) e Gomide-Junior (2013) que relataram $25,8 \%$ e $10,4 \%$ de pacientes com tratamento anterior, respectivamente, o que provavelmente se deve à melhor qualidade no preenchimento das fichas. Apenas em uma ficha esta informação foi negligenciada, não sendo possível afirmar se esse paciente recebeu este tipo de atendimento anteriormente, diferentemente dos estudos de Rigo e Honer (2005), que relataram essa falta de informação em 62,3\% dos atendimentos em Campo Grande (MS); e de Gomide-Junior (2013), que observou o não preenchimento do campo correspondente a essa informação em $31,6 \%$ das fichas.

Na presente análise, a espécie mais frequentemente relacionada a agressão aos humanos foi a canina, com 77,79\% dos casos, assim como em outros estudos (Ciampo et al., 2000; Araújo, 2002; Carvalho et al., 2002; Rigo \& Honer, 2005; Rolim et al., 2006; Poerner, 2007; Filgueira et al., 2011; Müller et al., 2011; Frias et al., 2012; Gomide-Junior, 2013; Silva et al., 2013; Benedetti et al., 2020; Marques et al., 2020). No mundo atual, os cães são os animais preferidos e mais presentes na sociedade humana como animal de companhia. Além desse fator, Buso et al. (2009) ressaltam que o maior número de agressões pela espécie canina nos serviços de saúde pode também estar relacionada ao melhor conhecimento por parte da população sobre o seu papel na cadeia epidemiológica como transmissor da raiva. O segundo animal mais envolvido em ataques a humanos em Jataí, foi o gato, muito embora em proporção bastante inferior à dos cães $(9,2 \%)$, situação encontrada também por outros autores (Rigo \& Honer, 2005; Müller et al., 2011; Frias et al., 2012; Macedo \& Silva, 2012; Gomide-Junior, 2013; Silva et al., 2013; Benedetti et al., 2020; Marques et al., 2020). Esse fato pode ser reflexo do comportamento felino, que apesar de ser um animal de companhia, tende a manter-se mais afastado dos seres humanos. Além disso, aponta-se um senso comum de considerar como grave apenas as agressões caninas, apaziguando arranhaduras por gatos (Carvalho et al., 2002). Os quirópteros são referidos como espécie agressora em número reduzido de casos $(0,71 \%)$ bem como nos resultados de GomideJunior (2013) que ressaltou o hábito noturno dessa espécie que os mantem distantes das pessoas. O contato desses animais com 
humanos, casualmente ocorre numa alteração de comportamento, que, incapacitado por morbidez, pode ser encontrado caído ou moribundo em locais não comuns (Reichmann et al., 2000).

Além de conhecer a espécie do animal agressor, é fundamental conhecer o seu estado de saúde para embasar a conduta médica no atendimento ao paciente agredido. Em Jataí, a maioria dos atendimentos foram provocados por animais sadios (86,32\%), fato também relatado por outros autores (Carvalho et al., 2002; Fortes et al., 2007; Poerner, 2007; Filgueira et al.,2011; Frias et al., 2012; Gomide-Junior, 2013). Ressalta-se que o estado de saúde dos animais é informado pela vítima e não necessariamente avaliada por um profissional capacitado, como um Médico Veterinário, e mesmo que a observação do animal agressor seja necessária para a correta conduta médica, ainda assim não existe um protocolo oficial para esse procedimento e a Ficha de Atendimento Antirrábica Humano não dispõe de campo para o parecer técnico.

Quanto ao período de observação da condição final do animal, dos 408 animais que foram observados $(81,27 \%), 402$ animais $(80 \%)$ deram negativo clinicamente para raiva e 6 animais $(1,27 \%)$ que tiveram amostras encaminhadas ao laboratório também apresentaram resultado negativo. Não houve resultado positivo para raiva tanto na parte laboratorial quanto clínica bem como não houve animais sacrificados com diagnóstico positivo para raiva, concordando com as descrições nos trabalhos de Veloso et al. (2011b) e Gomide-Junior (2013). Em relação aos 94 agravos (18,72\%) em que não houve observação do animal, o mesmo ocorreu por falha no acompanhamento pelos órgãos competentes, ou em situações em que o este não é passível de observação, como em casos de animais errantes que desaparecem logo após o acidente.

No que se refere a decisão médica quanto ao tratamento ao paciente atendido em Jataí, a observação do animal associado a vacinação pós-exposição, foi o tratamento médico mais adotado (78\%), assim como em outras análises (Veloso et al., 2011b; Frias, 2008; Poerner, 2007; Gomide-Junior, 2013). A conduta de apenas observar o animal doméstico ocorreu em 5,3\% dos casos, e está de acordo com os estudos de Carvalho et al. (2002); Filgueira et al. (2011); Müller et al. (2011) e Gomide-Junior (2013).

Os casos mais severos de agressão de animais às pessoas com maior probabilidade de exposição ao vírus rábico necessitam de terapêutica intensiva, com administração de soroterapia antirrábica, além da vacinação. No ano estudado, em Jataí, a soroterapia foi indicada em 13,3\% dos casos, proporção muito superior às relatadas por Garcia et al. (1999) com 0,05\%, Carvalho et al. (2002) com 2,4\%, Veloso et al. (2011b) com 6,4\% de indicações, na Grande São Paulo (SP), Maringá (PR) e Porto Alegre (RS), respectivamente; porém, igualmente ao relatado por Gomide-Junior (2013). Dessa forma, isso sugere a possibilidade de que a indicação de soro antirrábico em Luiz Antônio (SP) e em Jataí (GO) esteja sendo feita de modo excessiva e em não conformidade com as orientações oficiais, o que seria preocupante pelos riscos associados à administração do soro antirrábico e pelas suas limitações impostas aos receptores.

Tão importante quanto o tratamento indicado, é a efetiva realização do protocolo terapêutico. Dentre os atendimentos realizados em Jataí, o percentual de conclusão da profilaxia foi de 83,3\%, igualmente ao estudo de Gomide-Junior (2013), e ambos, superior ao estudo de Veloso et al. (2011b), que relatou mais de 50\% de interrupção por abandono em Porto Alegre (RS), e inferior à análise de Carvalho et al. (2002), que encontrou 92\% de tratamentos concluídos em Maringá (PR). Garcia et al. (1999) remeteram que baixas taxas de abandono decorrem de maior nível de conscientização da comunidade. Entretanto, Carvalho et al. (2002) concluíram que os abandonos ocorrem devido à perda de horas de trabalho, necessidade de acompanhantes, distância e despesas com transporte. Em Jataí, a maioria das interrupções de tratamento $(0,88 \%)$ ocorreu por indicação do próprio Serviço de Saúde e 0,53\% corresponderam a abandono por parte do paciente. Quando a interrupção do tratamento profilático antirrábico é caracterizada como abandono, o serviço de saúde deve proceder busca ativa para completar $\mathrm{o}$ atendimento do paciente (Gomide-Junior, 2013). Veloso et al. (2011b) reforçaram a ideia de que fica patente a necessidade do Serviço de Saúde aprimorar sua busca ativa de faltosos e melhorar sua sensibilidade quanto aos casos de abandono de 
tratamento; e ainda reforça que, duas estratégias devem ser utilizadas para enfrentar o abandono: capacitação da equipe de saúde e educação em saúde para a comunidade.

\section{Conclusão}

Diante do levantamento dos dados, constatou-se um elevada incidência de campos na Ficha de Atendimento Antirrábico com ausência de informações, ou preenchidos de forma incompletas e/ou incorretas, o que dificulta uma melhor análise da situação epidemiológica do atendimento antirrábico no município, e que colabora com a conclusão sobre a necessidade da capacitação da equipe de saúde para o completo e correto preenchimento das fichas individuais de atendimento e o empenho pela busca ativa dos faltosos ao tratamento.

Em relação a redução da profilaxia desnecessária, deve haver um aumento da observação dos animais e a integração entre os serviços de saúde médico e médico veterinário, uma vez o estado de saúde dos animais é informado pela própria vítima e não necessariamente avaliado por um profissional capacitado, como um Médico Veterinário.

Por fim, sugere-se uma constante atuação em pontos importantes para a manutenção do controle da raiva, quais sejam: a educação em saúde, a qualificação técnica dos profissionais que prestam o atendimento, e intensificação das ações de vigilância epidemiológica.

\section{Referências}

Araújo, F. A. A., (2002). Raiva humana no Brasil, 1992-2001 (Dissertação de Mestrado em Medicina Veterinária). Universidade Federal de Minas Gerais, Belo Horizonte, MG, Brasil.

Benedetti, M. S. G., Capistrano, E. R. S., Borges, M. G., \& Vieira Filho, J. (2020). Perfil epidemiológico dos atendimentos antirrábicos humanos no Estado de Roraima, Brasil. Brazilian Journal of Health Review, 3(5), 14017-14035. https://doi.org/10.34119/bjhrv3n5-211

Brasil. Ministério da Saúde (2014). Normas Técnicas de Profilaxia da Raiva http://portalarquivos.saude.gov.br/images/pdf/2015/outubro/19/Normas-tecnicas-profilaxia-raiva.pdf

Brasil. Ministério da Saúde (2017). Nota Informativa $\quad N^{o} \quad$ 26-SEI/2017-CGPNI/DEVIT/SVS/MS. http://portalarquivos.saude.gov.br/images/pdf/2017/agosto/04/Nota-Informativa-N-26_SEI_2017_CGPNI_DEVIT_SVS_MS.pdf

Buso, D.S., Nunes, C.M., \& Queiroz, L.H. (2009). Características relatadas sobre animais agressores submetidos ao diagnóstico de raiva, São Paulo, Brasil, 1993 - 2007. Cadernos de Saúde Pública, 25(12), 2747-2751. https://dx.doi.org/10.1590/S0102-311X2009001200021

Carvalho, W. O., Soares, D. F. P. P., \& Franceshi, V. C. S. (2002). Características do Atendimento Prestado pelo Serviço de Profilaxia Humana na Rede Municipal de Saúde de Maringá - Paraná, no ano de 1997. Informe Epidemiológico do SUS, 11(1), 25-35. https://dx.doi.org/10.5123/S010416732002000100004

Ciampo, L. A. D., Riccoa, R. G., Almeida, C. A. N., Bonilha, L. R. C. M., \& Santos, T. C. C. (2000). Acidentes de mordeduras de cães na infância. Revista de Saúde Pública, 34(4), 411- 412. https://doi.org/10.1590/S0034-89102000000400016

Filgueira, A. C., Cardoso, M. D., \& Ferreira, L. O. C. (2011). Profilaxia antirrábica humana: uma análise exploratória dos atendimentos ocorridos em Salgueiro-PE, no ano de 2007. Epidemiologia e Serviços de Saúde, 20(2), 233-244. https://dx.doi.org/10.5123/S1679-49742011000200012

Fortes, F. S., Wouk, A. F. P. F., Biondo, A. W., \& Barros, C. C. (2007). Acidentes por mordeduras de cães e gatos no município de Pinhais, Brasil, de 2002 a 2005. Archives of Veterinary Science, 12(2), 16-24. http://dx.doi.org/10.5380/avs.v12i2.9904

Frias, D. F. R. (2008). Avaliação dos registros de profilaxia antirrábica humana pós - exposição no município de Jaboticabal, São Paulo, no período de 2000 a 2006 (Dissertação de Mestrado em Medicina Veterinária Preventiva). Universidade Estadual Paulista, Jaboticabal, SP, Brasil.

Frias, D. F. R., Nunes, J. O. R., \& Carvalho, A. A. B. (2012). Caracterização de agravos causados por cães e gatos a seres humanos no município de Jaboticabal, São Paulo, durante o período de 2000 a 2009. Archives of Veterinary Science, 17(3), 63-70. http://dx.doi.org/10.5380/avs.v17i3.24824

Garcia, R. C. M., Vasconcellos, A. S., Sakamoto, S. M., \& Lopez, A. C. (1999). Análise de tratamento antirrábico humano pós-exposição em região da Grande São Paulo, Brasil. Revista de Saúde Pública, 33(3), 295-301. https://doi.org/10.1590/S0034-89101999000300011

Gomide Junior, M. H. (2013). Profilaxia da raiva humana em Luís Antônio, SP, Brasil: características das ocorrências e atenção médica prestada aos pacientes (Dissertação de Mestrado em Saúde na Comunidade). Universidade de São Paulo, Ribeirão Preto, SP, Brasil.

Macedo, L. P., \& Silva, P. L. N. (2012). Atendimento antirrábico humano: uma análise epidemiológica dos casos atendidos em 2010 na cidade de Montes Claros, MG. EFDeportes.com, Revista Digital, $15(166), \quad 1-1 . \quad$ https://www.efdeportes.com/efd166/atendimento-anti-rabicohumanoanaliseepidemiologica.htm 
Research, Society and Development, v. 10, n. 2, e13710212392, 2021

(CC BY 4.0) | ISSN 2525-3409 | DOI: http://dx.doi.org/10.33448/rsd-v10i2.12392

Marques, A. S., Silva Filho, A. G D., Carmo, D. M. D., Afonso, M. P. D., \& Oliveira, S. V. D. (2020). Profilaxia antirrábica no município de Uberlândia, Minas Gerais. Revista de Medicina da UFC, 60(2), 31-37. https://doi.org/10.20513/2447-6595.2020v60n2p31-37

Müller, G. C., Seger, J., \& Gabiatti, L.L. (2011). Avaliação dos casos de atendimento antirrábico humano notificados no município de São Miguel do Oeste SC no ano de 2009. Unoesc \& Ciência-ACBS, 1(2), 95-105.

Poerner, A. L. P. (2007). Tendência e características do atendimento antirrábico pós-exposição na região centro-sul fluminense, 2000 -2005 (Dissertação de Mestrado em Ciências). Universidade Federal Rural do Rio de Janeiro, Seropédica, RJ, Brasil.

Reichmann, M. L. A. B., Pinto H. B. F., Arantes, M. B., Santos M. B., Viaro, O., \& Nunes, V. F. P. (2000). Educação e promoção da saúde no Programa de Controle da Raiva. In: Manual Técnico do Instituto Pasteur. São Paulo: Instituto Pasteur, 30 pp.

Rigo, L., \& Honer, M. R. (2005). Análise da profilaxia da raiva humana em Campo Grande, Mato Grosso do Sul, Brasil, em 2002. Cadernos de Saúde Pública, 21(6), 1939-1945. https://doi.org/10.1590/S0102-311X2005000600044

Rocha, S. M., de Oliveira, S. V., Heinemann, M. B., \& Gonçalves, V. S. P. (2017). Epidemiological profile of wild rabies in Brazil (20022012). Transboundary and emerging diseases, 64(2), 624-633. https://doi.org/10.1111/tbed.12428

Rolim, R. L. P., Lopes, F. M. R., \& Navaro, I. T. (2006). Aspectos da vigilância epidemiológica da raiva no município de Jacarezinho, Paraná, Brasil, 2003. Semina: Ciências Agrárias, 27(2), 271-280. https://doi.org/10.5433/1679-0359.2006v27n2p271

Silva G. M., Brandespim D. F., Rocha M. D. G., Leite R. M. B., \& Oliveira J. M. B. (2013). Notificações de atendimento antirrábico humano na população do município de Garanhuns, Estado de Pernambuco, Brasil, no período de 2007 a 2010. Epidemiologia e Serviços de Saúde, 22(1), 95-102. http://dx.doi.org/10.5123/S1679-49742013000100010

Veloso, R. J., Aerts, D. R. G. C., Anjos, C. B., \& Sangiovanni, J. C. (2011a). Motivos de abandono do tratamento antirrábico humano pós-exposição em Porto Alegre (RS, Brasil). Ciência \& Saúde Coletiva, 16(2), 537-546. https://doi.org/10.1590/S1413-81232011000200017

Veloso, R. J., Aerts, D. R. G. C., Anjos, C. B., \& Sangiovanni, J. C. (2011b). Perfil Epidemiológico do atendimento antirrábico humano em Porto Alegre/RS, Brasil. Ciência \& Saúde Coletiva, 16(12), 4875-4884. https://doi.org/10.1590/S1413-81232011001300036 